\title{
Experiments on snap buckling, hysteresis and loop formation in twisted rods
}

\author{
V.G.A. Goss*, G.H.M. van der Heijden, J.M.T. Thompson, S. Neukirch
}

Centre for Nonlinear Dynamics, University College London, Gower Street, London WC1E 6BT, UK

November 5, 2004

\begin{abstract}
We give results of large deflection experiments involving the bending and twisting of $1 \mathrm{~mm}$ diameter nickel-titanium alloy rods, up to $2 \mathrm{~m}$ in length. These results are compared to calculations based on the Cosserat theory of rods. Details of this theory, formulated as a boundary value problem are presented. The mathematical boundary conditions model the experimental set up. The rods are clamped in aligned chucks and the experiments are carried out under rigid loading conditions. An experiment proceeds by either twisting the ends of the rod by a certain amount and then adjusting the slack, or fixing the slack and varying the amount of twist. In this way commonly encountered phenomena such as snap buckling, the formation of loops, and buckling into and out of planar configurations are investigated. The effect of gravity is discussed.

Key words: twisted rods, rod experiments, snap buckling, loop formation, snarling, hockling, welded boundary conditions.
\end{abstract}

*Current address: London South Bank University, Borough Road, London SE1 OAA, UK. 


\section{Introduction}

The distinctive feature of a thin rod is that it buckles rather than breaks when subjected to end forces and end moments. For example, experience tells us that a compressed rod can buckle in any lateral direction. It is also well-known that if a bending moment is applied at the ends of the compressed rod while in its buckled state, it may undergo a sudden large movement and "snap" into a configuration which is a mirror image of the buckled state. The symmetry associated with these buckling phenomena is a recurring theme in rod theory and is reflected in the mathematics where these types of phenomena are described by pitchfork bifurcation.

Perhaps not so well-known in rod mechanics is the existence of certain secondary bifurcations. These can be encountered by continuing to load the rod after it has buckled. They arise in a variety of industrial and biological applications. Amongst these we can mention "hockling" - the formation of loops during pipeline and cable laying operations. Hockling occurs when a twisted rod is slackened off and a section of it flips into a loop, [1], [2], [3], [4] and [5]. Other applications include "snarling", a term used in the textile industry [6] to describe the onset of highly twisted helical plies. More recently the mechanics of twisted rings as they jump in and out of various figure-of-eight configurations has been analysed [7], [8], a problem of specific relevance to the writhing of DNA filaments [9].

Many of these phenomena can easily be observed by twisting and bending a rubber rod with one's fingers, as shown in Figure 1. Experience teaches us that the response of a rod depends on the material it is made of, the geometry of its cross section, the manner in which it is held at its ends, the type of loading and the loading sequence. Over recent years a plethora of literature exploring, describing and explaining these effects has emerged, but very little in the way of experiments. This paper aims to help fill that gap.

The most straightforward experimental procedure for rod experiments is rigid loading. In this arrangement the displacements are controlled and the corresponding forces 


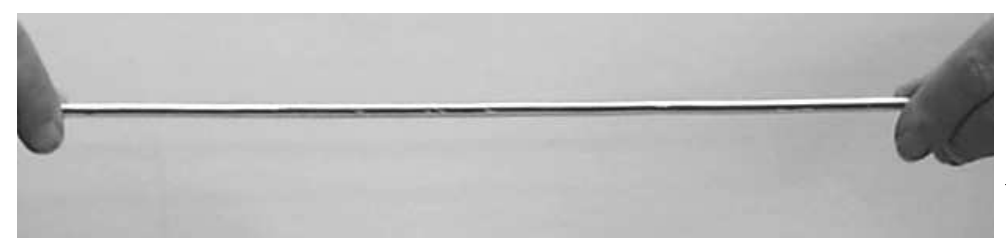

straight
untwisted rod

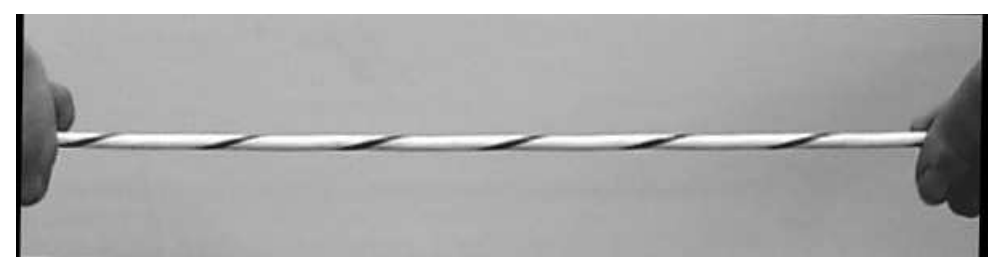

input twist

$(R)$

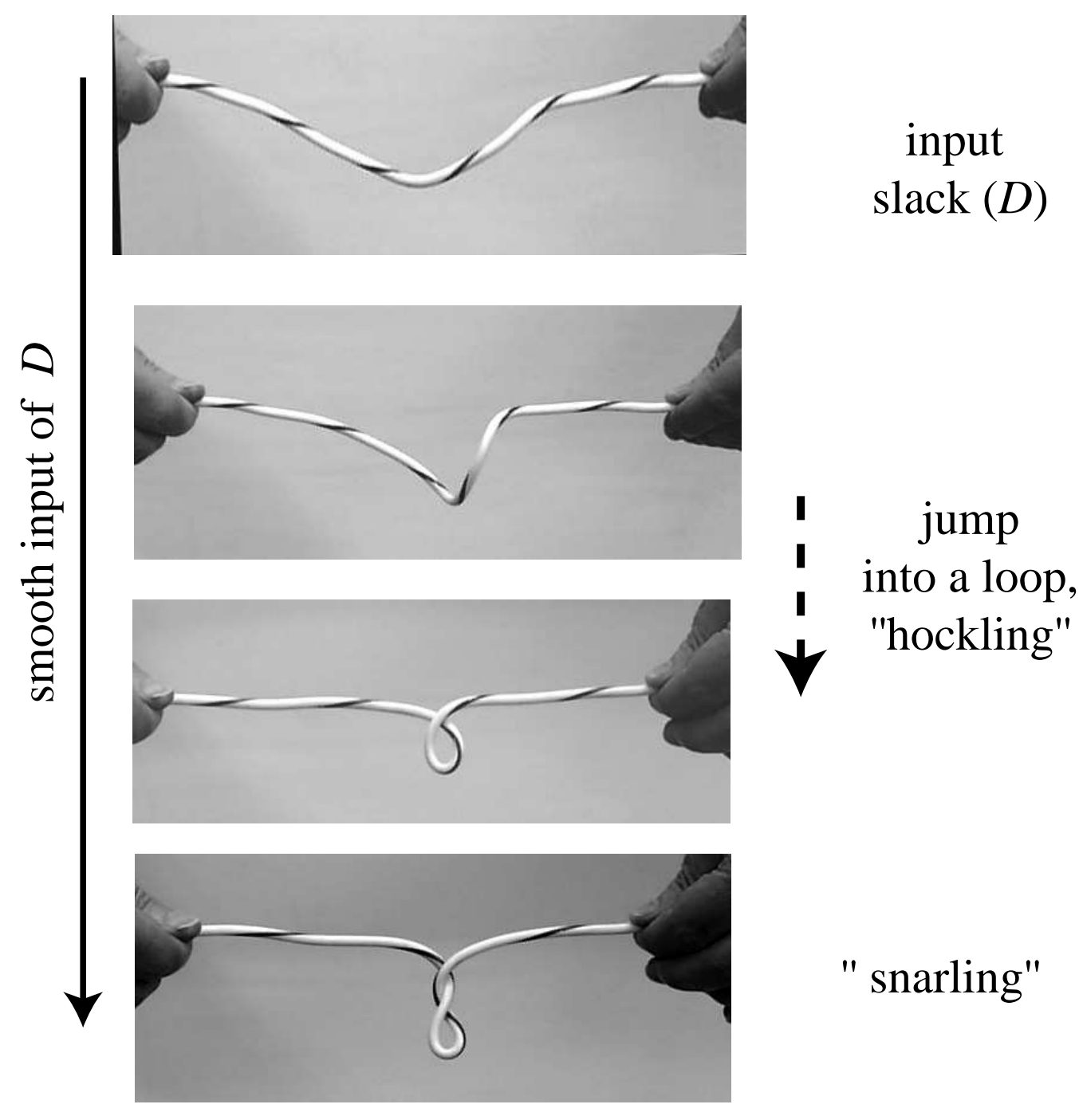

Figure 1: Slackening off a twisted rod (i.e., input of $D$ ) can cause it to throw a loop (hockling). If the initial twist is high enough, then further $D$ leads to snarling (i.e., the formation of a ply) 
and moments remain passive. The two pertinent control parameters are firstly, the displacement of the ends of the rod towards each other - the slack $D$; and secondly the rotation of one end of the rod with respect to the other end - the end rotation $R$. It is through $D$ and $R$ that the axial force $T$ (positive when tensile) and axial twisting moment $M$ do work respectively. Thus the natural arenas for the analysis of experimental results are $T D$ and $M R$ diagrams.

In his $\mathrm{PhD}$ thesis, Born [10] carried out some elegant large deflection bending experiments by hanging weights on the end of a rod (i.e., dead loading). Of the sparse experimental work carried out since, we can point to [2] and [5]. Using jacketed optical fibres Yabuta [5] carried out experiments in a rig with facilitation for measuring $R, D$, $M$ and $T$, and investigated both hockling and "pop-out" whereby the loop is removed by pulling it out (reversing $D$ ). However the precise manner by which the rods are fixed at their ends is not specified. Consequently, the mathematical model is not formulated as a boundary value problem. Instead, using an energy method, he assumes an initial helical deformation (which is Love's solution [11]) and obtained the Greenhill [12] formula for the onset of looping which in fact describes the primary bifurcation for a rod with zero bending moments at its ends. Modelling the loop as a circle, he also derived a formula for the point at which it reopens (i.e., pop-out) which he compared with his experimental results.

Thompson and Champneys [13] took up the problem of looping using modern geometric concepts of nonlinear dynamical systems theory. They reported on some qualitative experiments using silicone rubber rods which showed that if a highly twisted rod is slackened off it undergoes a localisation process prior to loop formation. In considering infinitely long rods, they identified the localised solutions as homoclinic orbits in the phase space of the underlying system of equations.

Miyazaki and Kondo [14] undertook theoretical investigations of finite rods. By choosing an Euler angle as the independent variable rather than arc length, they presented analytical solutions in a rather unwieldy integral form. Concentrating on two different rigid loading sequences, controlled $D$ with fixed $R$, and controlled $R$ with fixed 

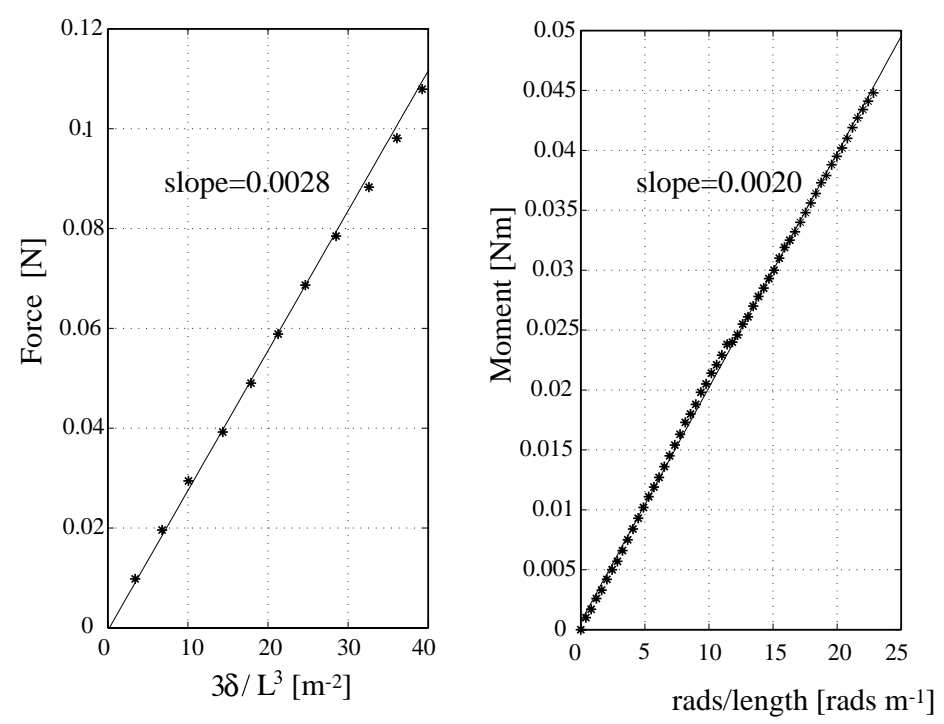

Figure 2: Best-fit line to experimental data for the determination of the flexural and torsional rigidities.

$D$, they give results for the onset of a number of secondary bifurcations, such as the formation of loops, snap buckling and bifurcations in and out of planar configurations.

However the work most closely associated with our experiments is [15] (which includes some of our experimental results), and should be referred to for the more analytical aspects of these investigations. This work deals with rods with isolated points of self contact, which we also briefly touch on in the experiments reported below.

This paper is set out as follows. Section 2 outlines the mathematical model with which we compare our results. We also give details of the experimental determination of the constitutive relations for the nitinol rods used in our experiments. In sections 3 and 4 we discuss and present results of experiments in which either $D$ or $R$ is fixed whilst the other is varied. Section 5 presents a discussion of our results including the effect of gravity. 


\section{Outline of the mathematical model}

\subsection{The Constitutive Relations}

Large deflection rod experiments have been hampered by the sometimes conflicting requirements that the rod possesses high flexibility, but that its statics is not dominated by sagging effects due to gravity; and that the loads are measurable. For one of these reasons or another, materials such as rubber, steel and nylon are not always convenient for experiments. However, the highly flexible nickel titanium alloy nitinol (which stands for Nickel Titanium Naval Ordnance Laboratory where it was first discovered by William Buehler in 1959 [16]) is a suitable material for a wide range of such experiments. Nitinol has the property that it undergoes a phase transition from the relatively ordered austenite solid-state phase to the more disordered martensite phase, either by varying (lowering) the temperature or stress-induced. The critical temperature can be varied from $100^{\circ} \mathrm{C}$ to well below $-200^{\circ} \mathrm{C}$, by changing the precise composition of the alloy.

Nitinol's high flexibility has been used in, for instance, spectacle frames, telecommunication antennae and actuator valves [17]. This flexibility together with its biocompatibility [18] is currently being exploited in a number of medical applications including surgical instruments, orthopaedic devices and orthodontic arches (see [19] for a recent review).

Nitinol rods of circular cross section with radius $r=0.5 \mathrm{~mm}$ and lengths $L$ varying from $300 \mathrm{~mm}$ to $2000 \mathrm{~mm}$ were selected. We assume, with good justification, that the specimens are isotropic and possess identical material properties at all points along their length (i.e., uniformity and homogeneity) and that their natural state is straight. We also assume that in our experiments they suffer no appreciable extension or transverse shear. It follows that we need only to establish the material's characteristic response to a lateral end force and a twisting moment: specifically the bending rigidity $B$ and the torsional rigidity $C$. Since suppliers advise that these properties vary with manufacturing history (especially heat-treatment), we established them directly 
by means of simple experiments. From a cantilever experiment the value of $B$ was determined using the engineer's formula ([20], page 369):

$$
\delta=\frac{P L^{3}}{3 B}
$$

where $\delta$ is the deflection measured at the free end of the rod due to the application of a known applied load $P$. As a consequence of isotropy, this experiment will give the same result in any lateral direction. To establish $C$, the rod was fixed at both ends and the torque measured for increments of end rotation $R$. The data from these measurements are shown in Figure 2. The straight line relationships between the loads and deflections indicate linear constitutive relations of the form:

$$
M_{1}=B \kappa_{1}, \quad M_{2}=B \kappa_{2}, \quad M=C \tau
$$

where $M_{1}$ and $M_{2}$ are the bending moments about two (orthogonal) principal axes of the cross-section (the reason for referring to principal axes will become clear in the next section), $\kappa_{1}$ and $\kappa_{2}$ are the respective curvatures, $M$ is the axial twisting moment, and $\tau$ is the twist per unit length. The slopes of the graphs in Figure 2 give $B=$ $0.0028 \mathrm{Nm}^{2}$ and $C=0.0020 \mathrm{Nm}^{2}$.

Using $B=E I$, where $E$ is the modulus of elasticity and $I=\frac{\pi r^{4}}{4}$ the second moment of area, we find $E=57 \mathrm{GNm}^{-2}$. This result is consistent with a separate tensile test carried out on a Tensometer 20 Universal Testing Machine. Quoted E values for nitinol alloys usually range from $28-44 \mathrm{Nm}^{-2}$ for the martensitic state and $80-110 \mathrm{GNm}^{-2}$ for the austenitic state [21]. However, our value correlates well with the range $32-60 \mathrm{GNm}^{-2}$ reported in [21] for heavily drawn wire.

Using $C=G J$, where $G$ is the shear modulus and $J=\frac{\pi r^{4}}{2}$ the polar moment of area, we find $G=20 \mathrm{GNm}^{-2}$. There is a scarcity of reported $G$ values in the literature. The only value of $G$ we could find is that extracted from experimental data reported in [22], which yields $G=21 \mathrm{GNm}^{-2}$. Our values imply a Poisson's ratio $\nu=\frac{E}{2 G}-1 \approx 0.4$.

Note that all measurements of forces and moments reported in this paper are in the 
range from -0.5 to $1 \mathrm{~N}$, and $\pm 0.2 \mathrm{Nm}$ respectively. The transducers used to measure the forces and torques have a sensitivity of $0.0001 \mathrm{~N}$ and $0.0001 \mathrm{Nm}$ respectively.

The constitutive relations (2) link the kinematics of a deformed rod to the equilibrium equations. These we treat in the following two sections. Together they establish the basis of the Special Cosserat Theory of Rods [23], a geometrically exact one dimensional theory, which we use to compare with our experimental results.

\subsection{The Kinematics}

Cosserat theory treats the rod as a set of material points forming a curve in space which we can think of as the centreline of the rod. The curve is parameterised by arc length $s \in[0, L]$ where $L$ is the length of the rod in its natural unstressed state, called the reference state. A distinctive feature of Cosserat theory is that it endows each material point with a unit vector $\mathbf{d}_{1}(s)$, called a director. The director is normal to the curve and aligned with a principal axis. The position of each point along the rod with respect to a fixed coordinate system (which is taken here to be coincident with the experimental rig, see Figure 3) is given by the vector $\boldsymbol{r}(s)=(x(s), y(s), z(s))$ with basis (i $\mathbf{j} \mathbf{k})$. The origin $\boldsymbol{r}(0)=(0,0,0)$ is taken to be at one end of the experimental rig, where the rod at $s=0$ is attached. For an unshearable, inextensible rod, we can define $\mathbf{d}_{3}$ as the tangent vector:

$$
\mathbf{d}_{3}:=\frac{\mathrm{d} \boldsymbol{r}}{\mathrm{d} s}
$$

We can then define $\mathbf{d}_{2}$ as follows:

$$
\mathbf{d}_{2}:=\mathbf{d}_{3} \times \mathbf{d}_{1}=\left(d_{3 y} d_{1 z}-d_{3 z} d_{1 y}\right) \mathbf{i}-\left(d_{3 x} d_{1 z}-d_{3 z} d_{1 x}\right) \mathbf{j}+\left(d_{3 x} d_{1 y}-d_{3 y} d_{1 x}\right) \mathbf{k},
$$

where the second suffix in each term of (4) refers to components in the fixed frame. The triad $\left(\mathbf{d}_{1}, \mathbf{d}_{2}, \mathbf{d}_{3}\right)$ constitutes a moving orthonormal frame attached to the rod. We assume that in its unstressed state the rod is straight and untwisted. We choose the directors in this reference state to be everywhere aligned with the rig axes, i.e., 


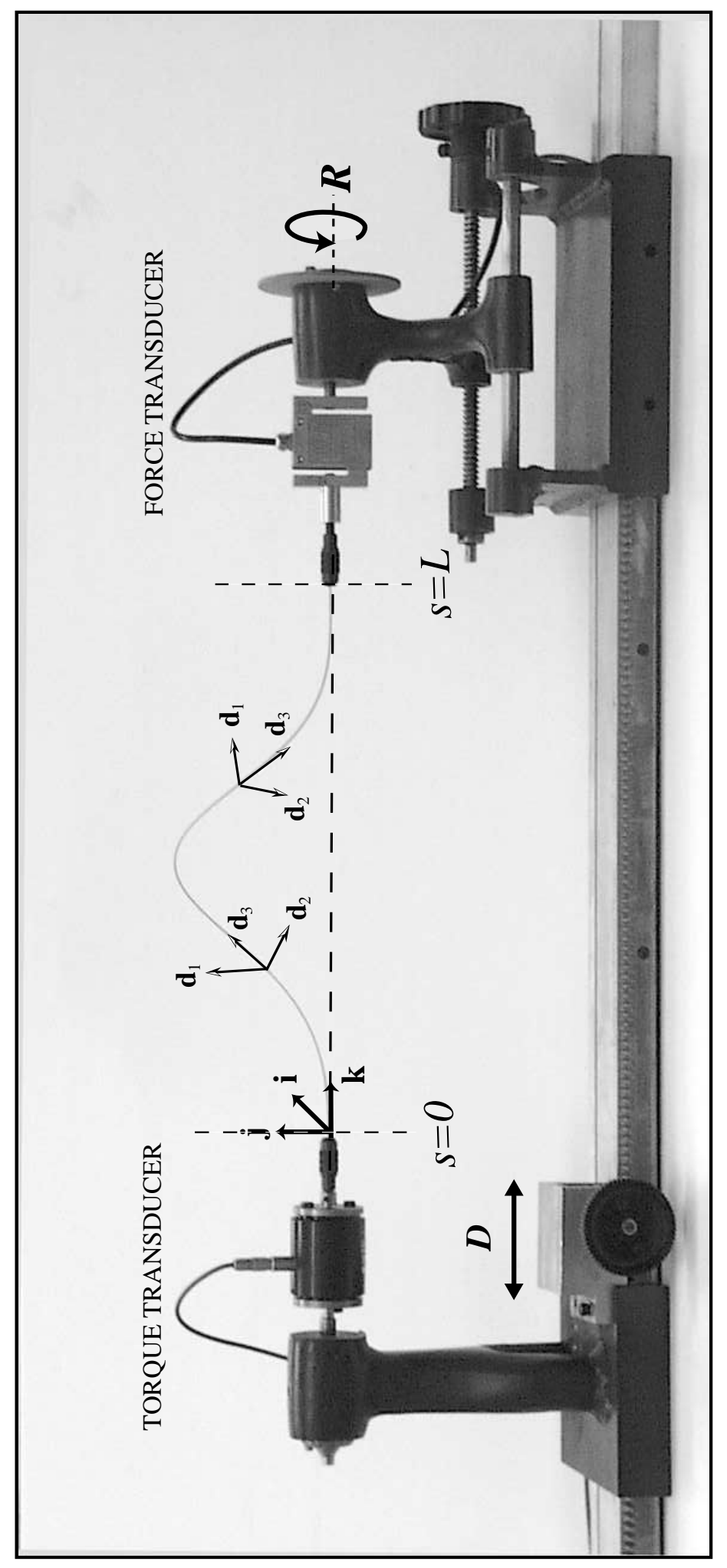

Figure 3: The experimental rig is designed as a rigid loading device whereby the slack $D$ and end rotation $R$ are controlled whilst the force $(T)$ and torque $\left(M_{3}\right)$ remain passive and are measured at the ends of the rod in the direction of $\mathbf{d}_{3}$. The photograph shows a length of nitinol rod with the directors $\mathbf{d}_{\mathbf{i}}$ superimposed. Also shown is the $\mathbf{i j k}$ rig-based frame. 
$\mathbf{d}_{1}=\mathbf{i}, \mathbf{d}_{2}=\mathbf{j}, \mathbf{d}_{3}=\mathbf{k}$. It follows that $\boldsymbol{r}=\mathbf{k} s$. Due to the orthonormality of the director frame, the spatial evolution of the directors is given as

$$
\frac{\mathrm{d} \mathbf{d}_{i}}{\mathrm{~d} s}=\boldsymbol{u} \times \mathbf{d}_{i} \quad(i=1,2,3),
$$

where $\boldsymbol{u}$ denotes the curvature vector

$$
\boldsymbol{u}=\kappa_{1} \mathbf{d}_{1}+\kappa_{2} \mathbf{d}_{2}+\tau \mathbf{d}_{3},
$$

$\kappa_{1}$ being the curvature about $\mathbf{d}_{1}, \kappa_{2}$ the curvature about $\mathbf{d}_{2}$ and $\tau$ the twist about $\mathbf{d}_{3}$.

\subsection{The Equilibrium Equations}

Newton and Euler's laws state that for statical equilibrium the resultant of the contact forces and contact moments must be zero [23]. Therefore, ignoring body forces, we have:

$$
\frac{\mathrm{d} N}{\mathrm{~d} s}=\mathbf{0},
$$

where $\boldsymbol{N}=N_{1} \mathbf{d}_{1}+N_{2} \mathbf{d}_{2}+N_{3} \mathbf{d}_{3}$, and $N_{1}$ and $N_{2}$ are shear forces and $N_{3}$ is the axial force (tension positive and compression negative). For the balance of moments we have

$$
\frac{\mathrm{d} \boldsymbol{r}}{\mathrm{d} s} \times \boldsymbol{N}+\frac{\mathrm{d} \boldsymbol{M}}{\mathrm{d} s}=\mathbf{0}
$$

where $\boldsymbol{M}=M_{1} \mathbf{d}_{1}+M_{2} \mathbf{d}_{2}+M_{3} \mathbf{d}_{3}$, and $M_{1}$ and $M_{2}$ are the bending moments and $M_{3}$ is the twisting moment.

The derivatives $\frac{\mathrm{d}}{\mathrm{d} s}$ on the left hand sides of (7) and (8) can be expressed as the derivative with respect to arc length in the moving co-ordinate frame, plus terms which describe the derivative of the directors themselves. For example: 


$$
\begin{aligned}
& \frac{\mathrm{d} \boldsymbol{N}}{\mathrm{d} s}=\frac{\mathrm{d}}{\mathrm{d} s}\left(N_{1} \mathbf{d}_{1}+N_{2} \mathbf{d}_{2}+N_{3} \mathbf{d}_{3}\right) \\
& =\left(\frac{\mathrm{d} N_{1}}{\mathrm{~d} s} \mathbf{d}_{1}+\frac{\mathrm{d} N_{2}}{\mathrm{~d} s} \mathbf{d}_{2}+\frac{\mathrm{d} N_{3}}{\mathrm{~d} s} \mathbf{d}_{3}\right)+\left(N_{1} \frac{\mathrm{d} \mathbf{d}_{1}}{\mathrm{~d} s}+N_{2} \frac{\mathrm{d} \mathbf{d}_{2}}{\mathrm{~d} s}+N_{3} \frac{\mathrm{d} \mathbf{d}_{3}}{\mathrm{~d} s}\right) .
\end{aligned}
$$

Consequently, using (5) and the constitutive relations (2), we can express (7) and (8) in component form:

$$
\begin{aligned}
\frac{\mathrm{d} N_{1}}{\mathrm{~d} s} & =\frac{N_{2} M_{3}}{C}-\frac{N_{3} M_{2}}{B}, \\
\frac{\mathrm{d} N_{2}}{\mathrm{~d} s} & =\frac{N_{3} M_{1}}{B}-\frac{N_{1} M_{3}}{C}, \\
\frac{\mathrm{d} N_{3}}{\mathrm{~d} s} & =\frac{N_{1} M_{2}}{B}-\frac{N_{2} M_{1}}{B}, \\
\frac{\mathrm{d} M_{1}}{\mathrm{~d} s} & =\frac{M_{2} M_{3}}{B}\left(1-\frac{B}{C}\right)+N_{2}, \\
\frac{\mathrm{d} M_{2}}{\mathrm{~d} s} & =\frac{M_{1} M_{3}}{B}\left(1-\frac{B}{C}\right)-N_{1}, \\
\frac{\mathrm{d} M_{3}}{\mathrm{~d} s} & =0 .
\end{aligned}
$$

An important property of the weightless isotropic rod is the existence of the following three independent constants:

$$
\begin{aligned}
& |\boldsymbol{N}|^{2}=N_{1}^{2}+N_{2}^{2}+N_{3}^{2}=\text { constant, } \\
& \boldsymbol{N} \cdot \boldsymbol{M}=N_{1} M_{1}+N_{2} M_{2}+N_{3} M_{3}=\text { constant, } \\
& \boldsymbol{M} \cdot \mathbf{d}_{3}=M_{3}=\text { constant. }
\end{aligned}
$$

The first two of (10) exist in the more general case of an anisotropic rod and correspond to the conservation of force and conservation of torque about the force vector. The line of action of this force vector is called the wrench axis. It is rotated and displaced 
with respect to the rig loading axis; see [15] for further details. The third constant is a consequence of isotropy and expresses conservation of twist about the rod's axis. Together the integrals in (10) render a fully integrable mathematical system of equations (see [24]) with solutions expressed in terms of elliptic integrals and elliptic functions. Whilst working with these integrals and functions has no benefit in the context of this experimental study (we use numerical methods), it is worth pointing out that the intrinsic symmetry in the mathematics manifests itself in the actual configuration of the rod: odd symmetry exists in the $z x$ plane, and even symmetry in the $z y$ plane. Photographs taken during the experiments have tried to capture this symmetry. Note though that strictly speaking the symmetry is with respect to the wrench axis, not the rig axis (see [15]).

\subsection{Boundary conditions and experimental procedure}

Since the rig is a rigid loading device we seek mathematical boundary conditions depicting the kinematics rather than the loads. First of all we specify the position vector at each end of the rod where it is attached to the rig:

$$
\boldsymbol{r}(0)=(0,0,0), \quad \boldsymbol{r}(L)=(0,0, L-D)
$$

where $D$ denotes the distance by which the end $s=L$ is displaced towards $s=0$ in a straight line along the $z$ axis, i.e., the slack. With respect to the tangent $\mathbf{d}_{3}$, the rig grips the ends of the rod in chucks in exactly the same way as a drill bit is gripped in a drill (see Figure 3). Consequently the slope at the ends is also fixed. Thus

$$
\mathbf{d}_{3}(0)=(0,0,1), \quad \mathbf{d}_{3}(L)=(0,0,1)
$$

Note that (11) and (12) correspond to Antman's welded boundary conditions [23]. Experiments may also involve inputting end rotation. When the rod is straight but twisted the rotation (modulo $2 \pi$ ) of one end with respect to the other end is given by $\mathbf{d}_{1}(0) \cdot \mathbf{d}_{1}(L)=\cos R$. Thus we have the following boundary conditions for $\mathbf{d}_{1}$ : 


$$
\mathbf{d}_{1}(0)=(1,0,0), \quad \mathbf{d}_{1}(L)=(\cos R, \sin R, 0) .
$$

We now have a system of fifteen first order ordinary differential equations; three in (3) for the position vector, six for the directors $\mathbf{d}_{1}$ and $\mathbf{d}_{3}$ in (5), and six in (9) for the forces and moments. Together with all nine boundary conditions stipulated at $s=0$ in (11), (12) and (13), plus three at $s=L$ in (11) and the conditions on $\mathbf{d}_{3 x}(L)$ and $\mathbf{d}_{3 y}(L)$ in (12) and $\mathbf{d}_{1 x}(L)$ in (13) constitute a well posed boundary value problem. This was solved by a single-parameter continuation of solutions using MATLAB's boundary value solver bvp4c. This is a finite difference code that implements a collocation formula, details of which can be found in [25]. Solution paths were traced out by gradually incrementing $D$ or $R$. We remark here that it is not always easy to find starting solutions in this continuation procedure and the program is slow when it is set at high accuracy.

Two different rigid loading experiments were carried out:

- Fixed $R$ loading: input $R$ and control $D$. These experiments are discussed in Section 3.

- Fixed $D$ loading: input $D$ and control $R$. These experiments are discussed in Section 4 .

In each set of these rigid loading experiments, the rig force $T$ and rig twisting moment $M$ are passive and are measured by the corresponding transducers at either end of the rod (see Figure 3). Because of the welded boundary conditions in which $\mathbf{d}_{3}(0)$ and $\mathbf{d}_{3}(L)$ are aligned with the rig, the transducers measure the axial loads and therefore $N_{3}=T$ and $M_{3}=M$. Each data point in the $T D$ and $M R$ diagrams to be presented represents the mean of at least three separate experiments taken at three separate times. Most experiments involved rods with lengths less than $\frac{1}{2} \mathrm{~m}$. 

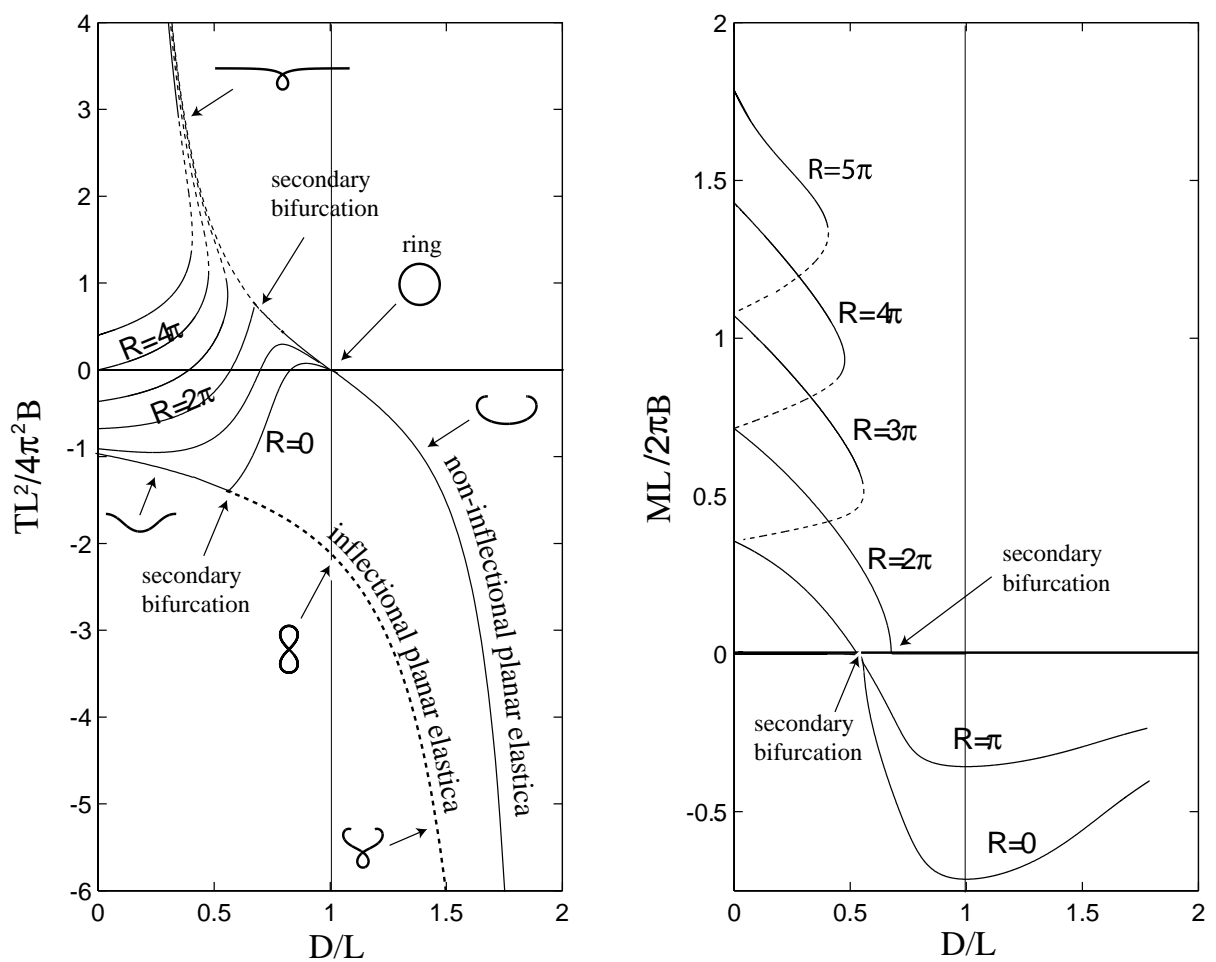

Figure 4: The theoretical $T D$ diagram and associated $M D$ diagram for rigid loading under control of slack. 


\section{Experimental results: $R$ fixed, $D$ varied}

It has already been mentioned that under fixed $R$ conditions the $T D$ diagram is the natural arena for the study of experimental results. Some insight into the global characteristics of the $T D$ diagram can be gained from consideration of the planar elastica - the two dimensional theory describing the planar configurations of a rod loaded only by an end force. This is a classical case in rod theory which Euler completely solved in 1744 [26]. The planar elastica solutions comprise two loading paths in the $T D$ diagram as shown in Figure 4. These two paths, the inflectional and noninflectional, represent a good guide to the upper and lower bounds of all other spatial loading paths for $D / L<1$.

In an experiment, a rod loaded only by an end force (i.e., $R=0$ ) buckles from the reference state at $T L^{2} / 4 \pi^{2} B=-1$ (Euler buckling). Under continued inputs of slack $D$ the rod bends in the plane and its loading path is initially identical to that of the inflectional planar elastica. However at a certain load a secondary bifurcation occurs. In the case of a nitinol rod this happens at $D / L=\Delta_{1}:=0.5590 \ldots$ [15]. In the $M D$ diagram this event is depicted by the appearance of nonzero twisting moments (see Figure 4). Thus this bifurcation involves a transfer of bending energy into twisting energy, causing the rod to twist out of the plane and depart from the inflectional planar elastica loading path. With further inputs of $D$ the rod gradually folds back upon itself until at $D / L=1$ it again adopts a planar state in the form of a twisted ring, having gained $2 \pi$ radians of twist.

Whilst our rig does not facilitate experiments in the vicinity of $D / L=1$, we can nevertheless conceive of a hypothetical experiment starting from a planar twist-free ring. We can proceed in one of two directions: either increasing or decreasing $D$. By increasing $D$ we open out the ring and follow the noninflectional planar elastica which remains stable for $D / L>1$. Experimental data for this case were obtained for $1.5 \leq D / L \leq 1.8$ and involve turning the rig ends around (see photograph in Figure $5)$. 

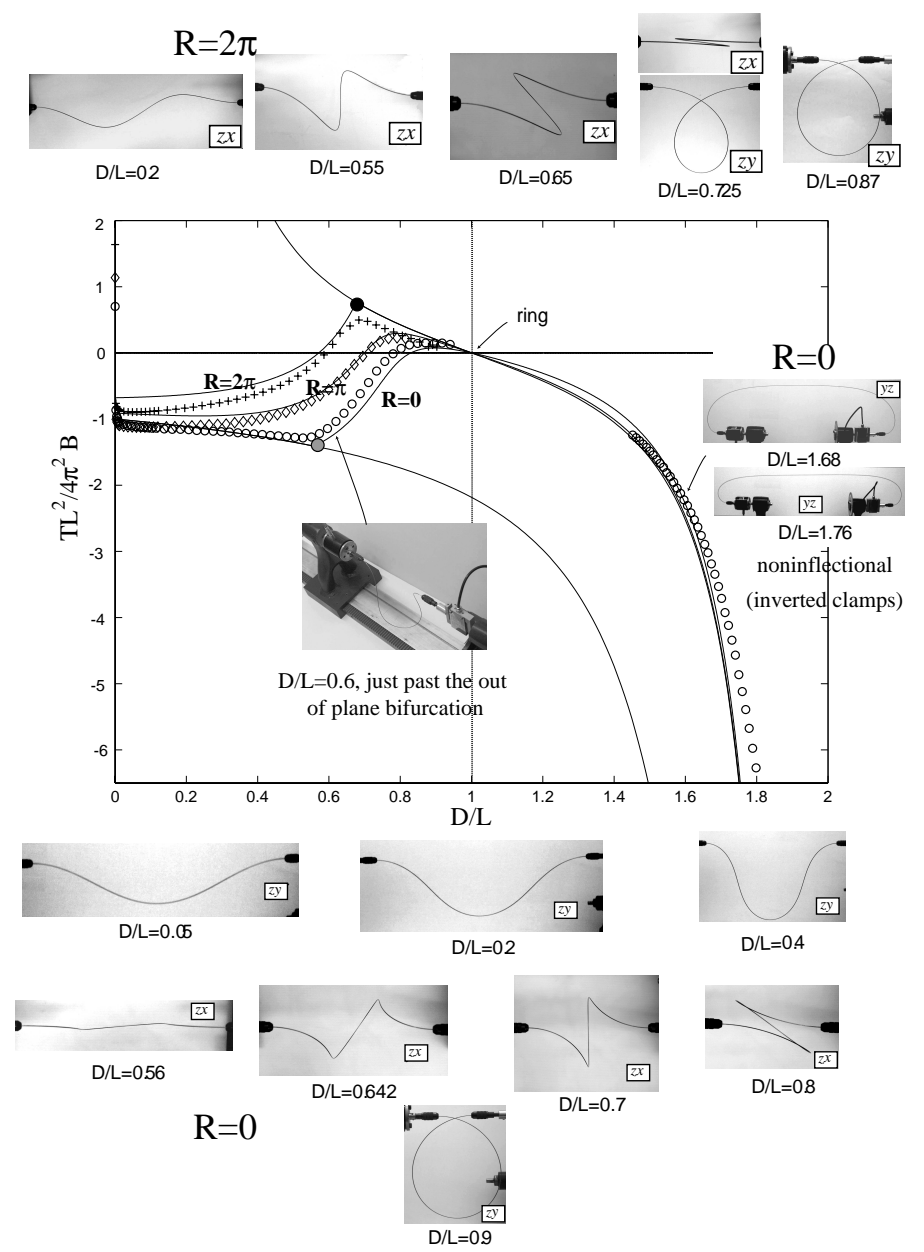

Figure 5: Experimental and theoretical results for rigid loading with end rotation fixed at $R=0$ (oоoo), $R=\pi(\diamond \diamond \diamond \diamond)$, and $R=2 \pi(+++++)$. For nitinol, with $\nu=0.4$, the bifurcation from the inflectional planar elastica (grey circle) occurs at a smaller value of $D / L$ than the bifurcation from the noninflectional planar elastica (black circle), but for other materials this can be reversed. Note the odd and even symmetries in the $z x$ and $z y$ planes respectively. 
By decreasing $D$ from the ring the ends of the rod are pulled across each other to form a loop. Whilst in this case the rod is strictly not planar (separated as it is by its cross sectional thickness) the loading path follows very closely that of the upper "self-intersecting" branch of the noninflectional planar elastica $(0<D / L<1)$; see Figure 5. In this case another secondary bifurcation arises $\left(D / L=\Delta_{2}:=0.6772 \ldots\right.$ for nitinol [15]) in which the rod twists out of the planar loop and buckles spatially. If the experiment is then continued all the way to the straight state at $D=0$, we find that the rod has again gained a twist of $2 \pi$ radians. We can also perceive this experiment in reverse: start at $D=0$ with $R=2 \pi$ and input slack until the rod bifurcates into the plane at $\Delta_{2}$. Further slack then retrieves the ring at $D / L=1$ with all the twist having been removed.

For $R \neq 0$ buckling is directly from a twisted straight state into a spatial configuration, mathematically described as a pitchfork bifurcation. The critical condition can be found from a linear eigenvalue analysis and is given in [15]:

$$
\frac{2 \pi n_{3}}{\lambda} \sin \pi \lambda=\cos \pi \lambda-\cos \pi m_{3}
$$

where $\lambda=\sqrt{m_{3}^{2}-4 n_{3}}$ with $m_{3}=\frac{C R}{B}$ and $n_{3}=\frac{T L^{2}}{4 \pi^{2} B}$. As more slack is input into the rod it releases twisting energy and transfers it to bending energy, adopting a 3D configuration. A characteristic of loading paths for $R<2 \pi$ is that they all lead to the planar ring at $D / L=1$, a state of zero tension (but non-zero twist).

For $R>2 \pi$ qualitatively different behaviour arises. A point is reached when the loading path develops a vertical tangency and changes direction (the knee of the curve in Figure 6). At this point the rod jumps off the path and flips into a self-contacting loop during which nearly $2 \pi$ radians of twist is released. Loop formation (hockling) is therefore described by a fold bifurcation and involves a transfer of twisting energy to bending energy. High initial end rotations are accompanied by correspondingly large jumps and occur at less slack.

Inspection of the $T D$ diagram in Figure 6 shows that hockling involves a jump towards the self-intersecting planar elastica loading path. If, after hockling, the slack 


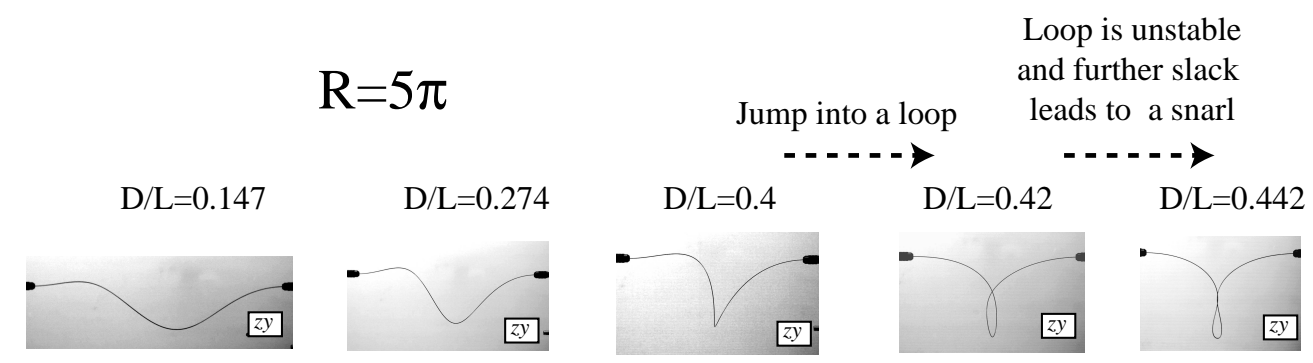

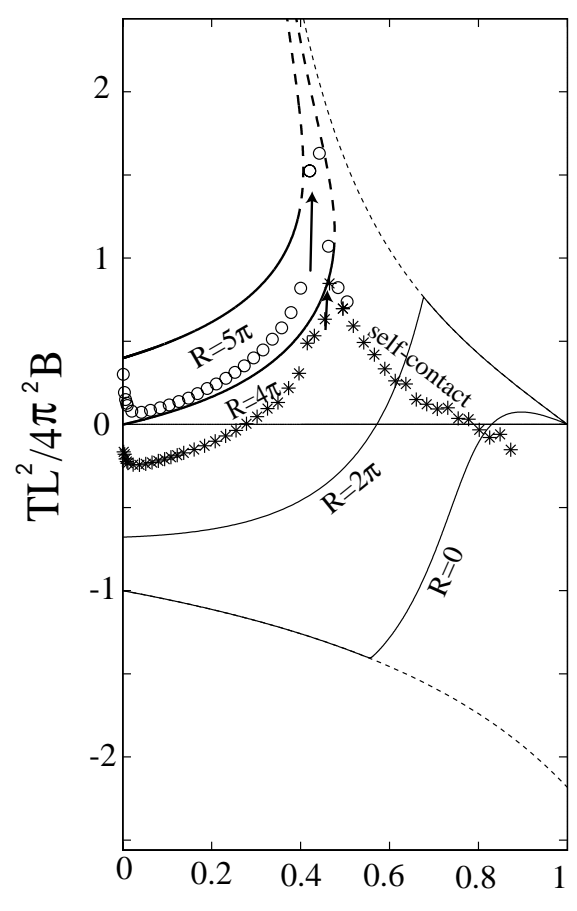

$\mathrm{D} / \mathrm{L}$

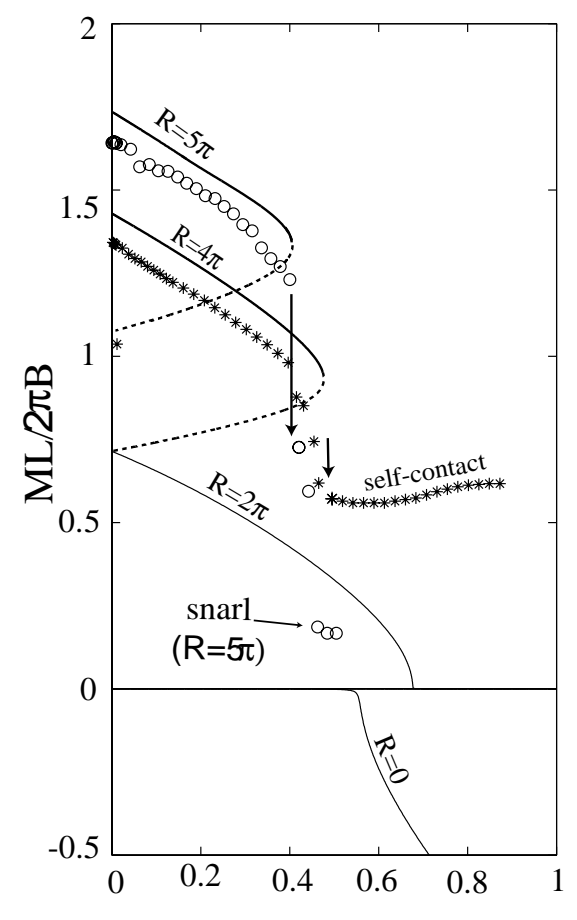

$\mathrm{D} / \mathrm{L}$

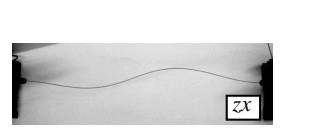

$\mathrm{D} / \mathrm{L}=0.05$

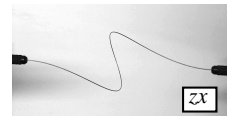
$\mathrm{R}=4 \pi$
$\mathrm{D} / \mathrm{L}=0.46$

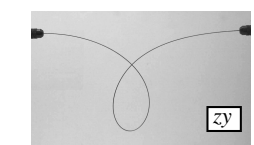

(2)

mp into a loop

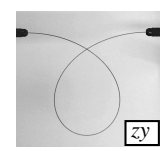

$\mathrm{D} / \mathrm{L}=0.648$

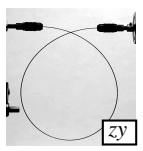

$\mathrm{D} / \mathrm{L}=0.86$

After the loop has formed it remains intact with further slack

Figure 6: $T D$ and $M R$ diagrams for $R=4 \pi$ and $5 \pi$. Note the presence of the hockling folds (vertical tangencies) on these loading paths. For $R>2 \pi$, smooth control of $D$ leads to a sudden jump into a loop when the path encounters one of these folds. In the case $R=4 \pi$ $(* * * *)$ the loop remains intact under increasing $D$ and forms a ring at $D / L=1$. However for $R>4.5 \pi$ the loop does not remain intact. Thus if $D$ is input after looping (see $R=5 \pi$ data (oooo)), the loop itself will rotate and form a ply (i.e., a "snarl"). 
is subsequently reversed back towards $D=0$, then the loop is under tension and pulled tight, causing an increase in curvature, and therefore the bending moment, around the loop. A point is reached when this configuration becomes unstable and the loop pops out. In the $T D$ diagram pop-out corresponds to the rod falling back onto its original loading path, thereby creating an hysteresis cycle. Note that the path to pop-out may be accompanied by plastic deformation resulting in a permanently damaged kinked rod. This occurs when the loop dimensions decrease to the point that the curvature becomes so large as to push the rod outside of its linearly elastic regime.

On the other hand, increasing D after loop formation can cause the rod to "snarl", i.e., form a ply, as shown in Figure 6, which may also damage the rod. The onset of snarling is only evident beyond a critical value of $R$, and for nitinol this was found experimentally to be at $R \approx 4.5 \pi$ radians, a result consistent with the theoretical amount of end rotation that can be input into a ring before it buckles spatially, given in $[27]$ :

$$
R_{\text {crit }}=2 \pi \sqrt{3}(1+\nu)
$$

which for nitinol gives $R=4.85 \pi$ radians. For $R$ less that this value the loop remains intact and is converted into a twisted ring at $D / L=1$.

\section{Experimental results: $D$ fixed, $R$ varied}

In this section we discuss experiments on rods that are loaded by twisting the ends relative to each other whilst $D$ is fixed. Five qualitatively different regions of behaviour are identified, and their demarcation depends upon the critical values $\Delta_{1}$ and $\Delta_{2}$ at which the secondary bifurcations arise. Numerical data for loading paths within each region are shown in Figure 7. We now discuss our experimental results, treating each of these regions in turn.

- $D / L<\Delta_{1}$

The two distinctive characteristics of this region are that firstly, since $D / L<\Delta_{1}$, the planar elastica is stable and consequently the rod can be rotated through 


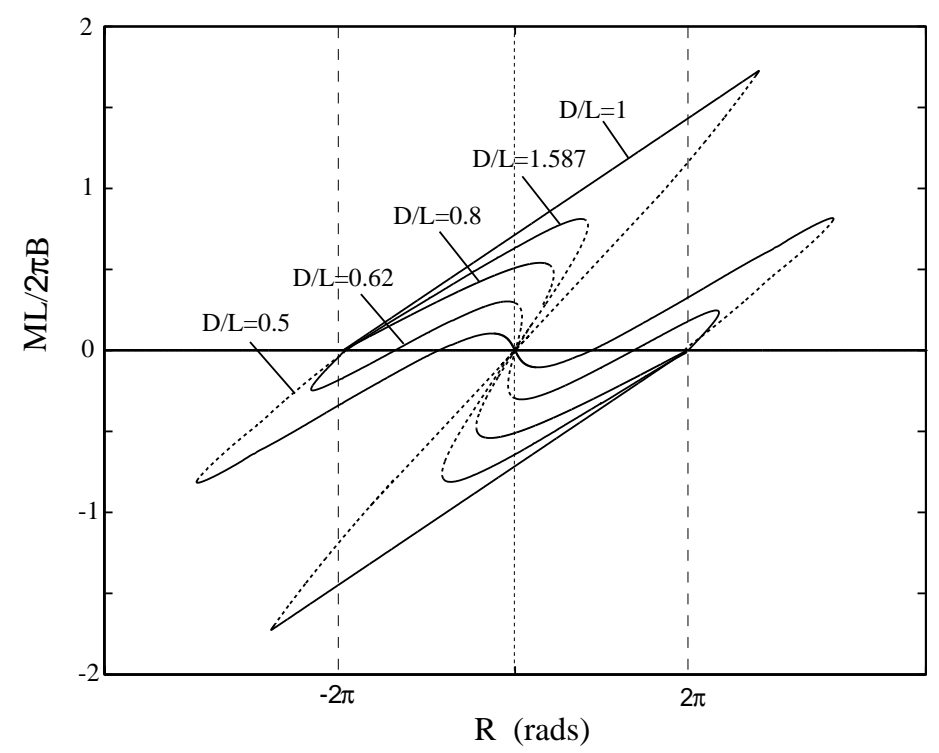

Figure 7: The theoretical $M R$ loading diagram, for a range of fixed $D$ values. Note that the maximum twisting moment occurs in the $D / L=1$ case. For $D / L>1$ the moment reduces again.

$R=0$ smoothly; and secondly, a jump into a loop occurs once $R>2 \pi$, corresponding to hockling. The loop can be removed by unwinding $R$ to induce pop-out. The paths between hockling and pop-out form an hysteresis cycle, as shown in Figure 8. Note that the theoretical path through the self-contact data between $B$ and $C$ in this figure is provided by the (friction-free) point contact theory in [15].

- $\Delta_{1}<D / L<\Delta_{2}$

Once $D / L$ exceeds the value $\Delta_{1}$ the planar state is no longer stable and the rod jumps through $R=0$, a phenomenon known as snap buckling, which is described mathematically as a pitchfork bifurcation. Under further input of $R$ the rod will jump into a loop (hockling) which can be removed by unwinding $R$ (pop-out). These phenomena are shown in Figure 9 where it can be seen that both snap buckling and hockling involve hysteresis cycles. Whilst the amplitude of the jump associated with snap buckling rises under increasing $D$, there is an 


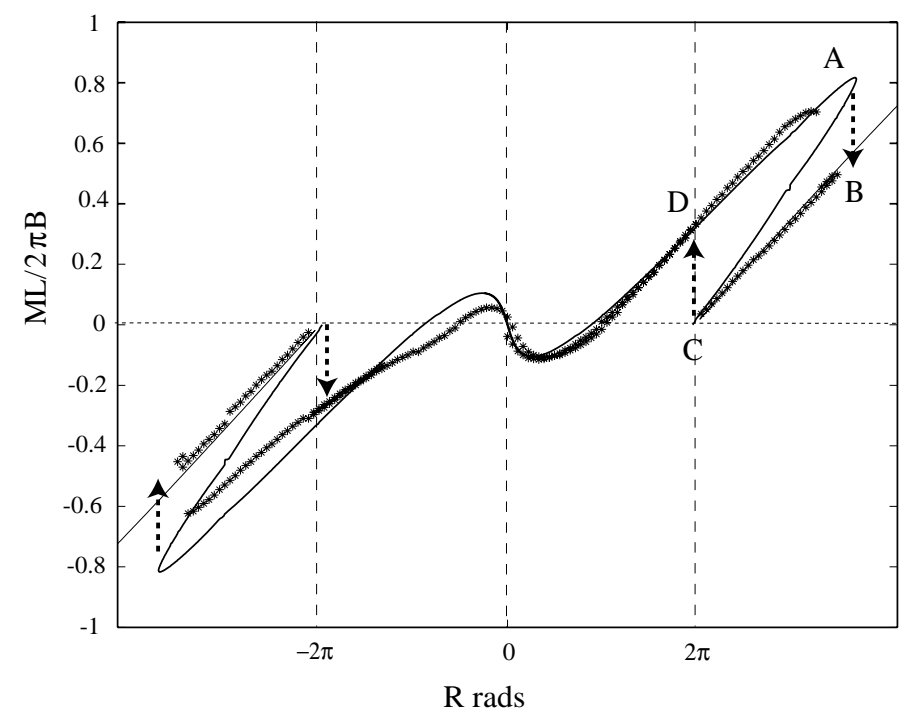

Figure 8: Results of an experiment conducted under control of $R$, with slack fixed at $D / L$ $=0.5$. The rod passes smoothly through $R=0$ but becomes unstable at $\mathrm{A}$ and jumps into a loop (to B). Reversing $R$ causes the rod to pop out of the loop $(\mathrm{C})$ and return to the stable branch at D (without self-contact). The circuit ABCD forms an hysteresis cycle, also encountered in negative $R$. We observe that the fit is better in positive $R$ than in negative $R$, but we can offer no explanation for this.

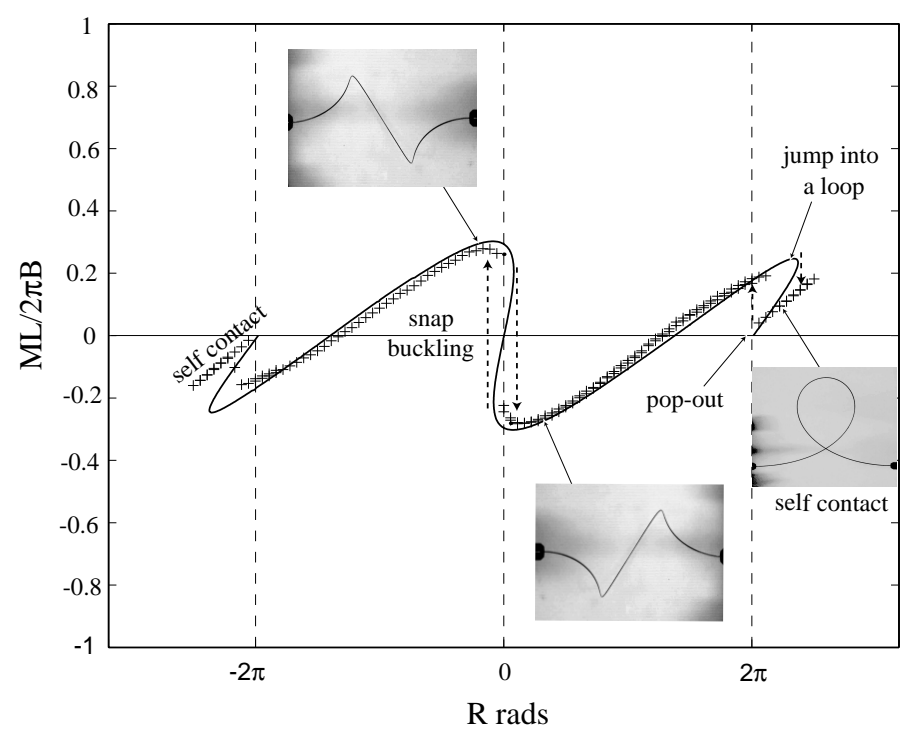

Figure 9: Experimental and theoretical $M R$ results for $D / L=0.62$, showing snap buckling, looping, pop-out and the hysteresis cycles. 
accompanying decrease in the magnitude of the jump into and out of self-contact during hockling (see Figure 10).

- $\Delta_{2}<D / L<1$

When $D / L$ exceeds the value $\Delta_{2}$ the jump to self-contact and its attending hysteresis loop disappear and are replaced by a smooth path (see Figure 10). However the magnitude of the snap buckling jump continues to increase.

- $D / L \approx 1$

At $D / L=1$ a rod forms a closed ring. A small but distinctive region of ringlike behaviour exists in its vicinity. Figure 11 plots the theory for $D / L=1$ and experimental results for $D / L=0.98$ (with the rod attached directly to the torque transducer).

In the experiment the amount of twist that the ring absorbs before it becomes unstable is consistent with (15). The rod then jumps from its twisted planar state into a "pinched" figure of eight, see Figure 11). Swigon's analysis [7] predicts that the ply forming the central part of this configuration has two points of self contact, implying the existence of a gap in between. However no gap was discernable in the experiment (see lower photograph in Figure 11). Unwinding $R$ brings about another state: at $M \approx 0$ the rod jumps into a figure-of-eight with one cross over point (consistent with [7]). After further reduction of $R$ the rod jumps back to a twisted ring, again when $M \approx 0$.

- $D / L>1.2$

Experiments for $D / L>1$ were conducted with the clamps turned around. When $R$ is input into the rod whilst lying in a plane with $D / L \approx 1.2$, a section towards the end of it is seen to rotate out of the plane, but not to a condition of self contact. Note however that this does not imply the nonexistence of stable selfcontacted solutions for $D / L>1$ (see Figure 10 of [15]). 

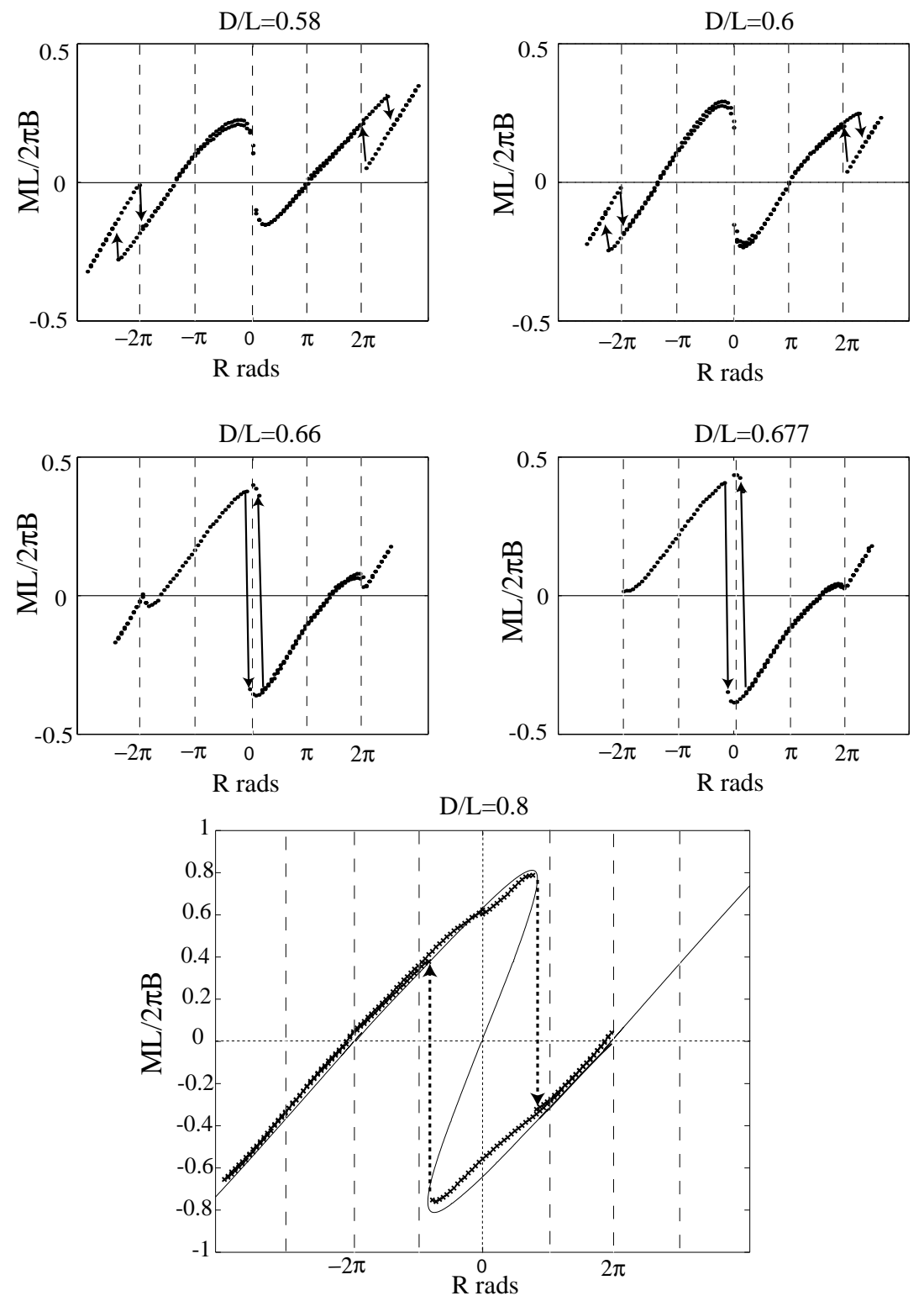

Figure 10: $M R$ diagrams showing experimental data obtained under control of $R$ with $D / L$ fixed. The top four figures show cases where the value of $D / L$ lies between the two secondary bifurcations, i.e., $\Delta_{1}<D / L<\Delta_{2}$. Note that for each increase in $D / L$ the jump associated with snap buckling (which occurs in the vicinity of $R=0$ ) is more pronounced whilst the jump associated with hockling (which occurs when $R>2 \pi$ ) decreases in magnitude and eventually disappears at $D / L=\Delta_{2} \approx 0.6772 \ldots$. For $D / L>\Delta_{2}$ loop formation occurs smoothly and does not involve a jump (see lower figure with $D / L=0.8$ ), but the magnitude of the snap buckling jump continues to increase. 

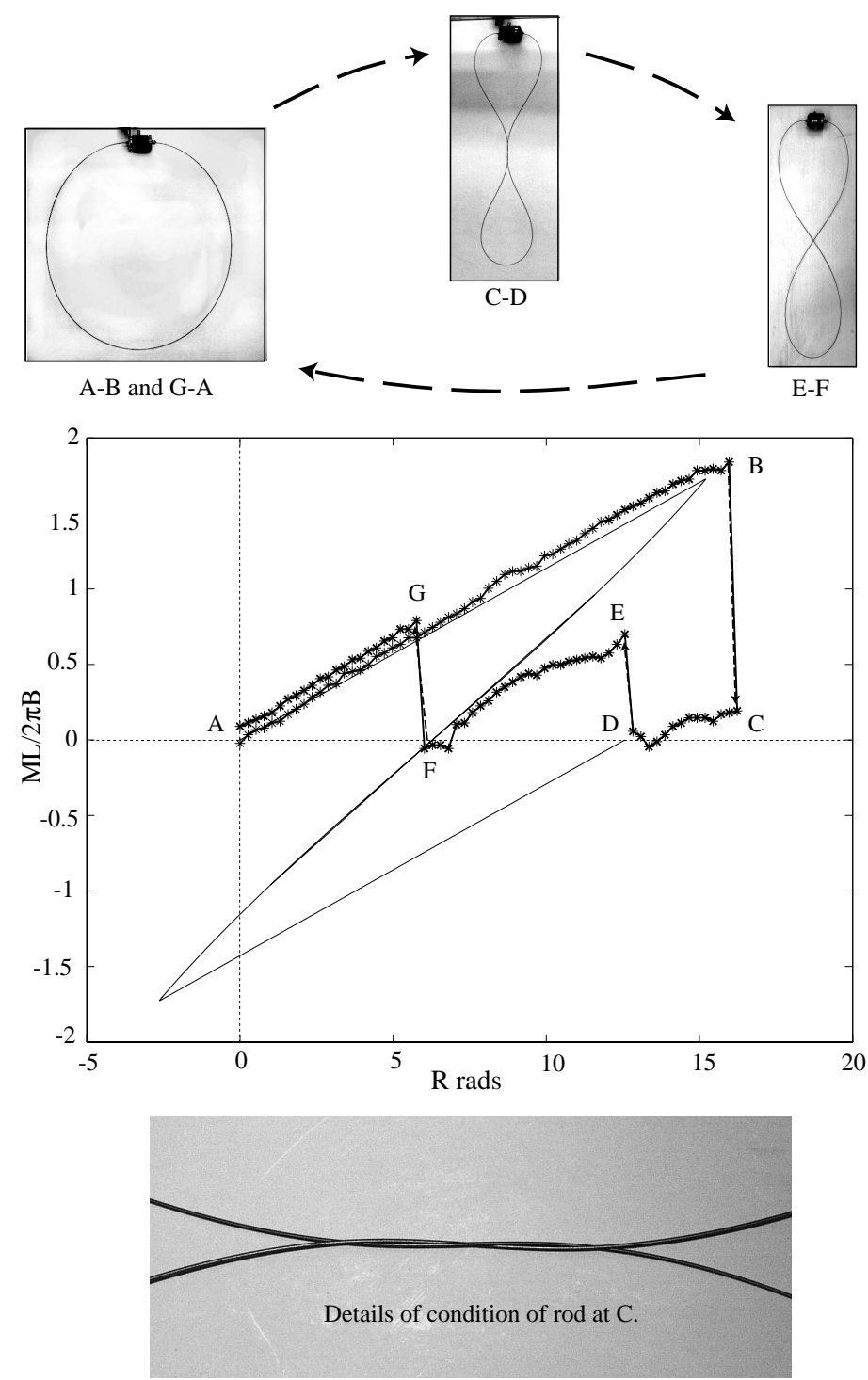

Figure 11: $M R$ diagram for an experiment in which end rotation is input into the rod with $D / L=0.98$, where it is in the form of a ring. The twisted ring remains stable until the loading path reaches B at which point it jumps into a "pinched" figure of eight. Subsequent reduction of $R$ induces a jump ( $D \longrightarrow E$ ) into a figure of eight with a single point of selfcontact. Further unwinding induces another jump $(F \longrightarrow G)$, this time back on to the original loading path where it remains until $R$ is reduced to zero and we retrieve the twist-free ring at $\mathrm{A}$. 


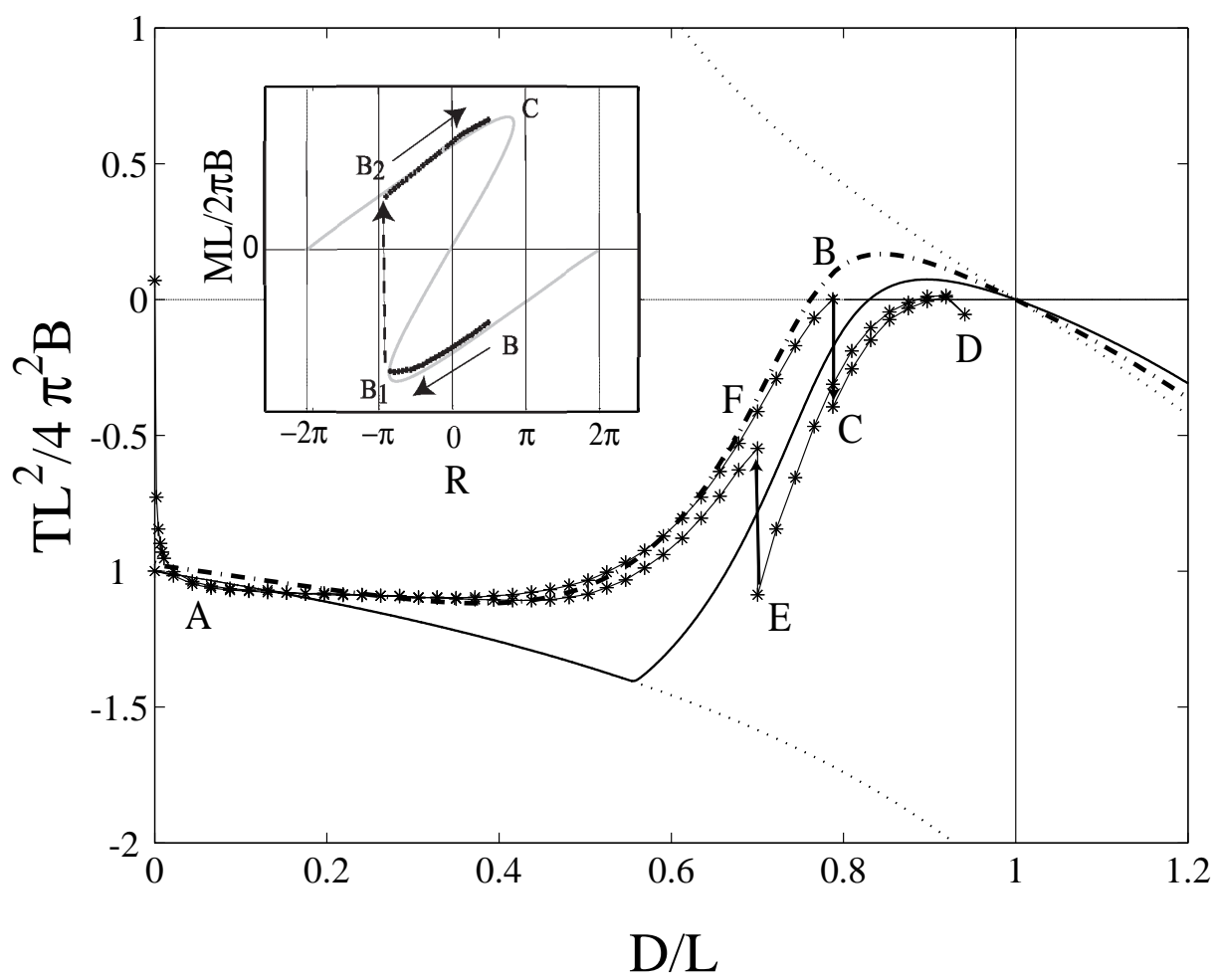

Figure 12: Mixed loading experiment. The experimental $(* * * * *)$ and theoretical $(-\cdot-\cdot)$ $T D$ loading paths for $R=\pi / 2$. The experiment is initiated from $A$ under control of slack until point $B$ (which lies beyond the secondary bifurcation at $\Delta_{1}$ ). The experiment is then paused and placed under control of $R$ with $D$ fixed (see $M R$ diagram in the inset). Snap buckling $\left(B_{1} \longrightarrow B_{2}\right)$ causes the rod to jump across the $R=0$ curve $(B \longrightarrow C$ in the $T D$ diagram). After winding $R$ back to its original value of $\pi / 2\left(B_{2} \longrightarrow C\right.$ in the $M R$ diagram), the experiment is resumed under control of slack $C \longrightarrow D \longrightarrow E$, at which point the loading path encounters a fold, and the rod spontaneously jumps back across the $R=0$ path to $F$. Further reduction of slack takes the rod on a smooth path all the way back to $A$. 
It is worthwhile to include in our investigation mixed loading sequences, in which both $D$ and $R$ are varied. Under special circumstances it is possible to encounter unexpected instabilities. Figure 12 gives the results of an experiment which initially proceeds under control of slack with $R=\pi / 2$. The experiment is momentarily stopped at a point when the slack exceeds $\Delta_{2}$, and therefore the planar elastica is unstable. The experiment is continued under control of $R$. Adjusting $R$ induces snap buckling which lands the rod on a new path which is situated in a region of the $T D$ diagram beyond the $R=0$ path, a region which is not accessible under sole control of $D$. It reveals an interval of $D / L$ values with more than one stable solution, a phenomenon which arises as a consequence of the nonlinearity of our system; the reader should refer to [15] for a more thorough investigation of the multiplicity of solutions for the elastic rod equations. With $R$ wound back to $\pi / 2$ and the experiment resumed under control of $D$ the rod undergoes another bifurcation (by encountering a fold on the new path) and snap buckles back across the $R=0$ path to its original path.

\section{Discussion}

The focus of our numerical study is the statics of an inextensible, unshearable, uniformly isotropic, weightless Cosserat rod. Here we can be assured by its quintessence. However, in treating the Cosserat rod as a reference point for the study of the behaviour of real rods as observed through experiment, we need to pay special attention to the experimental set-up. As far as is practically possible, we strive to identify and eliminate those physical imperfections which may cause an experiment to deviate from the mathematical model. For example we require rods with linear elasticity at large strains, and avoid heavy rods. Our experiments indicate that deviation between experiment and Cosserat theory is likely to arise in situations where the strains are high and where friction may arise, such as in states with high twist and self-contact. We note here that in an attempt to keep the twist per unit length such that the rod remains in a linearly elastic regime, long rods were used for high twist experiments. We also note 
the sensitivity of experimental results to boundary conditions.

A noticeable qualitative discrepancy between our fixed $R$ experimental results and the theory is a rounding off of the primary bifurcation, given by (14). This is only evident in the $T D$ diagrams, particularly in Figure 6 , and indicates the presence of an imperfection which does not effect the twisting moment. An obvious source of this error is therefore the rod's self weight. We also remark that a heavy rod would be expected to buckle at a higher tension than a light rod.

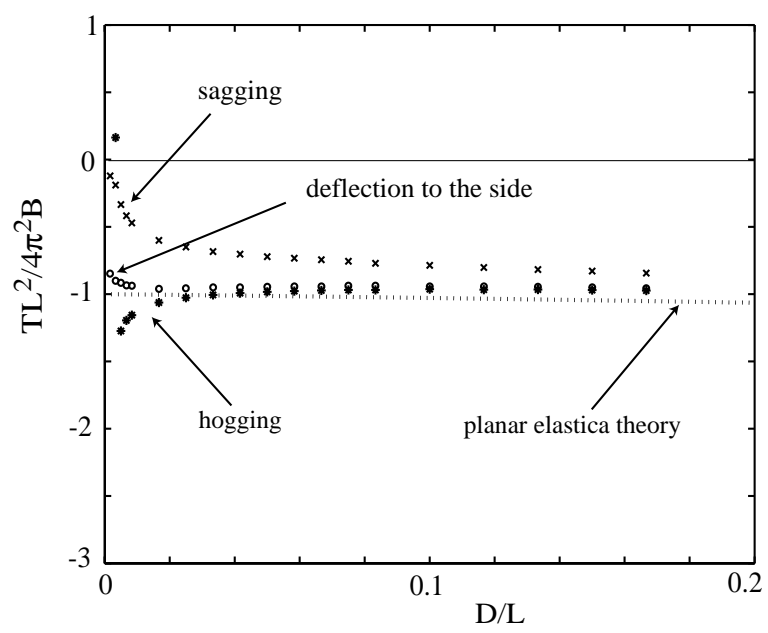

Figure 13: Experiments on a rod with a rectangular cross section $(3 \mathrm{~mm} \times 0.5 \mathrm{~mm} \times 600$ $\mathrm{mm})$ can give different results in the magnitude of the buckling force. This difference is due to rod's weight and depends on whether it buckles by sagging, hogging (i.e., rising upwards) or by deflecting to one side. Sagging results in a steep negative gradient, hogging results in a positive gradient and for the case that the rod buckles to one side, the effect of gravity is minimised.

The effect of self weight was investigated by performing experiments on a $600 \mathrm{~mm}$ long rod with a flat cross section (i.e., a tape). Tapes can be easily induced to bend in the plane perpendicular to their flat side, whether this is up (hogging), down (sagging) or sideways. Experimental data corresponding to these three different modes are shown in Figure 13, where it can be seen that a tape, initially held under tension, which buckles by hogging delays the primary bifurcation, whilst sagging hastens it. A difference of $4 \mathrm{~mm}$ between the maximum $x$ deflection in these two modes was recorded. Force 
diagrams which include weight, imply that as the angle of deflection $\theta$ increases (due to inputs of $D$ ), a tape which sags is under higher tension than a tape which hogs, but the difference declines as slack increases.

Weight can be incorporated into the mathematical model by replacing (7) by $\frac{\mathrm{d} \boldsymbol{N}}{\mathrm{d} s}=m(s) g \mathbf{k}$, where $m$ is the mass per unit length, which from measurements is $0.0048 \mathrm{~kg} / \mathrm{m}$. Preliminary numerical results show that the inclusion of the rod's weight leads to a rounding off of the primary bifurcation as observed in our experimental data. However the magnitude of the offset between experiment and theory is insignificant and does not reflect the quantitative offset between the gravity free theory and some of our experimental results, especially those at high fixed $R$ (see Figure 6). It is likely that our assumptions of linear constitutive relations and nonshearability break down as $R$ increases. We note here that experiments were particularly sensitive to errors in the boundary conditions (12).

This work shows that if a loop forms in a rod, then unwinding the twist may instigate a dynamic jump as the rod pops out of self contact. Therefore to ensure smooth removal of loops, the slack should be increased beyond the out-of-plane bifurcation before reversing $R$. Note that by trying to pull the loop out by reversing the slack may damage the rod.

\section{References}

[1] Coyne, J., "Analysis of the formation and elimination of loops in twisted cable," IEEE Journal of OCEAnic EngineERIng 15 (2), 72-83 (1990).

[2] Liu, F.C., "Kink formation and rotational response of single and multistrand electromechanical cables," Technical Note N-1403, Civil Engineering Lab, Naval Construction Batallion Centre, Port Hueneme, California (1975).

[3] Rosenthal, F., "The application of Greenhill's formulae to cable hockling," ASME Journal of Applied Mechanics 43, 681-683 (1976). 
[4] Tan, Z. and Witz, J.A., "Loop formation of marine cables and umbilicals during installation," in Proceedings of Behaviour of Offshore Structures, 1992, London (BOSS '92), Vol. II, M.H. Patel and R. Gibbins (eds), 1270-1285 (BPP Technical Services, London, 1992).

[5] Yabuta, T., "Submarine cable kink analysis," Bulletin of the Japenese SoCIETy of Mechanical EngineERS 27 (231), 1821-1828 (1984).

[6] Hearle, J.W.S. and Yegin, A.E., "The snarling of highly twisted monofilaments," Journal of the Textile Institute 63 (9), 477-489 (1972).

[7] Swigon, D., "Configurations with self contact in the theory of the elastic rod model for DNA," PhD dissertation, Rutgers State University of New Jersey, USA (1999).

[8] Coleman, B.D. and Swigon, D., "Theory of supercoiled elastic rings with self contact and its application to DNA plasmids," Journal OF ElAsticity 60, 173-221 (2000).

[9] Calladine, C. R. Drew, H. Luisi, B. Travers,A., Understanding DNA: The Molecule and How It Works, 3rd ed, Elsevier Academic Press, London, (2004).

[10] Born, M., "Untersuchungenüber die Stabilität der elastischen linie in Ebene und Raum," PhD thesis, University of Göttingen, Germany (1906).

[11] Love, A.E.H., A Treatise on the Mathematical Theory of Elasticity, 4th ed, Cambridge Univerity Press, (1927).

[12] Greenhill, A.G., "On the strength of shafting when exposed both to torsion and to end thrust," Proceedings of the Institute of Mechanical Engineers LONDON, 182-209 (April 1883).

[13] Thompson, J.M.T. and Champneys, A.R., "From helix to localised writhing in the tosional post-buckling of elastic rods," Proceedings of the Royal Society of London, A452, 117-138 (1996). 
[14] Miyazaki, Y. and Kondo, K., "Analytical solution of spatial elastica and its application to kinking problem," International Journal of Solids and StrucTURES 34 (27), 3619-3636 (1997).

[15] Van der Heijden, G.H.M. Neukirch, S. Goss, .V.G.A. and Thompson, J.M.T., "Instability and contact phenomena in the writhing of clamped rods," InTERNAtional Journal of Mechanical Sciences 45, 161-196 (2003).

[16] Kauffman, G.B. Mayo, I., "The story of nitinol: The serendipitious discovery of the memory metal and its applications." The Chemical Educator 2 (2), 121 (1996).

[17] Pemble, C.M. Towe, B.C., "A miniature memory alloy pinch valve" SEnsors AND ACTUators 77, $145-148$ (1999).

[18] Kujala, S. Pajala, A. Kallioinen, M. Pramila, A. Tuukkanen, J. Ryhanen, J. "Biocompatibility and strength properties of nitinol shape memory alloy suture in rabbit tendon," Biomaterials 25, 353-358, (2004).

[19] Kusy, R.P., "Orthodontic Biomaterials: From the Past to the Present," Angle Orthodontist 72, (6), $501-502$ (2002).

[20] Gere, J.M. and Timoshenko, S.P., Mechanics of Materials, 2nd ed, Van Nostrand Reinhold (International) Co. Ltd. London (1987).

[21] Rucker, B.K. Kusy, R.P., "Elastic properties of alternative versus single strand ed leveling archwires," American Journal of Orthodontics And DentofaCIAL ORThopedics 122 (5), 528-541 (2002).

[22] Drake, S.R. Wayne, D.M. Powers, J.M. Asgar., "Mechanical Properties of orthodontic wires in tension, bending, and torsion," AmERican Journal OF ORTHODONTICS 82 (3), 206-210 (1982).

[23] Antman, S.S., Nonlinear Problems of Elasticity, Springer-Verlag, New York (1995). 
[24] Kehrbaum, S. and Maddocks, J.H., "Elastic bodies, quarternians and the last quadrature," Proceedings of the Royal Society of London. A355, 2117-2136 (1997).

[25] Shampine, L.W. Kierzenka, J. and Reichelt, M.W., "Solving boundary value problems for ordinary differential equations in MATLAB with bvp4c." ftp://ftp.mathworks.com/pub/doc/papers/bvp/ (2000).

[26] Euler, L., "Additamentum 1 de curvis elasticis, methodus inveniendi lineas curvas maximi minimivi proprietate gaudentes," Bousquent, Lausanne (1744). Reprinted in Opera Omnia I 24, 231-297.

[27] Zajac, E.E., "Stability of two planar loop elasticas," TransaCtions of THE ASME Journal of Applied Mechanics 29, 136-142 (1962). 